3 Running title: Spatiotemporal variation in functional redundancy

4 Authors: Aoife Cantwell-Jones ${ }^{1}$, Keith Larson ${ }^{2}$, Alan Ward ${ }^{1}$, Olivia K. Bates ${ }^{1}$, Tara Cox $^{1}$, Frida

5 Brannlund ${ }^{2}$, Charlotte Gibbons ${ }^{1}$, Ryan Richardson ${ }^{1}$, Jason M. Tylianakis ${ }^{3}$, Jacob Johansson ${ }^{4}$ \& Richard

6 J. Gill ${ }^{1}$

\title{
7 Contributions:
}

$8 \mathrm{ACJ}, \mathrm{AW}$ \& RJG conceived the idea; KL initiated the phenology project; $K \mathrm{~L}, \mathrm{OKB}$, TC \& RJG set up the 9 permanent study site and developed the research goals; KL, OKB, TC, FB, CG, RR \& RJG performed

10 the fieldwork; ACJ, AW \& RJG developed the analytical approach, with feedback from JT and JJ; ACJ

11 performed the analyses with input from AW \& RJG; ACJ \& RJG wrote the manuscript, with feedback

12 from KL, JT \& JJ.

\section{Addresses:}

14 1. Georgina Mace Centre for The Living Planet, Department of Life Sciences, Silwood Park, Imperial

15 College London, SL5 7PY, UK

17 University, 90187 Umeå, Sweden. 


\section{Acknowledgements}

$25 \mathrm{ACJ}$ is funded by the NERC Science and Solutions for a Changing Planet doctoral training programme,

26 Imperial College London. JMT is funded by the Marsden Fund (Grant number: UOC1705). The project

27 was also supported by an INTERACT grant (funded by H2020 - agreement no. 730938) awarded to

28 RJG. We would like to thank J. Gustafsson for help with plant identification, H. Rosenzweig for help

29 setting up the sampling plots, and the Climate Impact Research Centre (CIRC, Umeå University) \&

30 Abisko Scientific Research Station (ANS) for field equipment and their continued support.

31 Competing interests: The authors declare no competing interests.

32 Data statement: All data used in this study will be uploaded to the Dryad Digital Repository on

33 acceptance.

34 Word count:

35 Abstract: 150 words

36 Main: 5,000 words

37 Figures: 5

38 Tables \& Boxes: 0

39 References: 103 


\section{ABSTRACT:}

41 Functional overlap between species (redundancy) shapes competitive and mutualistic interactions,

42 determining community responses to perturbations. Most studies view functional redundancy as

43 static, even though individuals within species vary in traits over seasonal or spatial gradients.

44 Consequently, we lack knowledge on trait turnover within species, how functional redundancy

45 spatiotemporally varies, and when and where interaction networks are vulnerable to functional loss.

46 Studying an Arctic bumblebee community, we investigated how body-size turnover over elevation

47 and season shapes their host-plant interactions, and test how sensitive networks are to sequentially

48 losing body-size groups. With trait turnover being larger than species, we found: i) late-season

49 networks were less specialised when nodes comprised functionally similar bumblebees; ii) removal

50 of bumblebee-body-size groups over species accelerated coextinction of host plants, with the

51 magnitude varying in space and time. We demonstrate functional redundancy can vary

52 spatiotemporally, and functional loss impacts interaction partners more than expected from species

53 loss alone. 


\section{1. INTRODUCTION}

56 Understanding the processes structuring communities over environmental gradients is central to

57 determining how they will respond to environmental change (Pellissier et al., 2018; Orr et al., 2021).

58 In particular, our ability to predict how organisms interact through trophic, mutualist or competitive

59 processes depends on being able to map how functional traits vary in space and time, both within

60 and between species (Gray et al., 2018; Gravel et al., 2019; Gómez et al., 2020; Hurtado et al., 2020).

61 While key life-history traits directly determine inter- or conspecific relationships and the distribution

62 of individuals, such traits are also simultaneously being selected and filtered by the environment

63 (Gillespie et al., 2017; Bladon et al., 2020). Consequently, the frequency distributions of traits within

64 populations can vary across environmental gradients and over seasons (Jourdan et al., 2016; Boyle et

65 al., 2020; Taylor-Cox et al., 2020; Gutiérrez \& Wilson, 2021). Yet how population-level responses

66 combine to influence community trait-composition, and subsequently how spatiotemporal trait

67 turnover feeds back to shape community interactions, remain poorly understood (Ings et al., 2009;

68 Tylianakis \& Morris, 2017; Peralta et al., 2020).

For insect pollinators, how functional traits are spatiotemporally distributed likely determines the

70 occurrence of interactions with mutualistic host plants (Bewick et al., 2013; Brosi \& Briggs, 2013),

with compatibility between pollinator traits and plant hosts mediating interaction strengths

72 (Bartomeus et al., 2016; Peralta et al., 2020). Investigating the dynamics of plant-pollinator

relationships, however, has often been based on network comparisons between sites separated by 
al., 2020), and understand how species versus functional traits spatiotemporally turn over in

81 localised communities (Robroek et al., 2017). In doing so, we can resolve the contribution of

82 functional traits to network structure and robustness to perturbation. With trait variation

responding to many environmental factors, studies mapping the distribution of trait variability can

more accurately reveal how environmental change can shape and potentially threaten plant-

pollinator interactions.

Bipartite networks can investigate how pollinator communities interact with their host plants by given trait from a set of sampled individuals (e.g. Bartomeus, 2013; Coux et al., 2016; Dehling et al., 2016). This assumes however that all individuals within each node are functionally equivalent and overlap in trait values between species is low - which is rarely the case (Gentile et al., 2021). et al., 2014), with intraspecific trait variation potentially being as large as interspecific (Des Roches et al., 2018), arising through sexual dimorphism (Bolnick et al., 2011), conditions during development (Fenberg et al., 2016) and adaptation to environmental gradients (Hurtado et al., 2020; Taylor-Cox et al., 2020). Additionally, temporal intraspecific variation can result from phenological variation with members of a species emerging at different times (Dongmo et al., 2018; Fric \& Konvicka, 2002). must scale down to the functional traits of individuals within populations, to disentangle how traits shape networks (e.g. Dupont et al., 2014; Kuppler et al., 2016; Rumeu et al., 2018). To our 
Here, we explore how individual variability in traits and interactions contribute to spatiotemporal intraspecific trait variability will be sufficiently large that functional turnover in space and time will exceed species turnover; (2) interaction networks where pollinator individuals are grouped together into nodes based on their traits, rather than species identity, will better capture interacting guilds of space and time.

We test these hypotheses in a montane Arctic community of bumblebees, using bumblebee-plant visitation data collected repeatedly along an elevational transect ( 33 days) over a 57-day period. This temporal and elevational cline allowed us to map plant-bumblebee interaction and trait turnover in space and time. We focus on body size, as it is influenced by thermal conditions during development, and determines foraging capabilities at low and high relative temperatures (Lundberg \& Ranta, 1980; Greenleaf et al., 2007). Body size also strongly correlates with proboscis length

123 (influencing floral compatibility; Cariveau et al., 2016) and can predict pollinator-plant interactions 124 (Eklöf et al., 2013; Klumpers et al., 2019). Additionally, as with many functionally important species, bumblebee body size at the population level does not remain constant through the season, as earlier emerging individuals have larger bodies (queens) than later emerging individuals (workers).

\section{METHODS}


130 We collected data along an elevational transect (420-1164 m.a.s.I.; est. 1917; Fries, 1925;

131 MacDougall et al., 2021) on the eastern slope of Mt Nuolja in Abisko National Park, Sweden (68 $22^{\prime}$

$\left.132 \mathrm{~N}, 18^{\circ} 47^{\prime} \mathrm{E}\right)$. Along the $3.4 \mathrm{~km}$ transect, there are 13 permanent plots measuring $45 \times 45 \mathrm{~m}$, within

133 which we recorded bumblebee activity (Fig. 1 \& S1; SI 1). Five relatively distinct vegetational zones

134 (Fig. 1 \& S2; Table S1) are found along this transect and following Lundberg \& Ranta (1980) can be

described as: A) old Birch forest (420-550 m; $n=3$ plots); B) young, newly established Birch forest

(550-650 m; $n=2)$; C) shrub Willow (650-850 m; $n=3)$; D) herbaceous meadow (850-1050 m; $n=2)$;

E) Arctic dwarf shrub and heath $(1050-1164 \mathrm{~m} ; n=3)$. The number of plots $(n=13)$ was determined

by the feasibility under which sampling could take place, dependent on both the terrain and number

of observations that could be achieved in one day.

\subsection{Field observations and body size measurements}

We conducted observations between $24^{\text {th }}$ May to $20^{\text {th }}$ July 2018 , weather permitting. We typically conducted a "survey" - defined as undertaking bumblebee observations for all 13 plots over a twoday period (intended to be two consecutive days) - twice per week (Table S1). We observed each plot for a standardised 20 minutes between 08:00 - 19:00. For each survey, we randomised the time of day the plot was sampled (SI 2 for further details).

We recorded all bumblebees observed flying through or foraging inside the plot $(n=1,582)$, but only included those that were confidently identified taxonomically in the analyses (13 species; $n=1,047$ individuals). If the bee was foraging on a flower, we recorded the plant species ( $n=31 ;$ Table S2). We attempted to catch all observed bees with a butterfly net (45 cm diameter), and, if successful, transferred them ( $n=709$ ) into separate lidded, plastic holding pots (150 mL volume) and placed them in a dark insulated bag. At the end of the observation period, we transferred each bee in order of capture to a marking cage and took a top-down dorsal image using a digital camera (Canon SX720 HS) once the bee was still (e.g. Fig 1, inset B). From the images, we: i) confirmed species 
indistinguishable (Williams et al., 2015) and therefore grouped as a species aggregate; ii) measured

the intertegular distance (ITD; distance between left and right forewing attachment points, which is

an accurate proxy for body mass (Cane, 1987) and proboscis length (Cariveau et al., 2016)), by

digitally landmarking each tegulum using TPS software (tpsDig v.2.3.2 \& tpsUtil v. 3.2; Rohlf, 2015)

and exporting the landmarks to the geomorph package in $\mathrm{R}$ to calculate scaled distance in

millimetres (Adams et al., 2020). Of the bees with ITD measurements, we observed $69.8 \%(n=495)$

foraging on a flower, and these comprised 94 queens, 270 workers, 21 drones, and 110 females

where caste could not be confidently assigned (Table S3-5; SI 3).

\subsection{Assessing intra- and interspecific variation in bumblebee body size}

To gauge the magnitude of intraspecific variation in body size, we quantified the degree of overlap in

ITD distributions between species by calculating the "coefficient of overlapping" (Ridout \& Linkie,

2009) of pairwise bumblebee species kernel density estimates using the overlapTrue function of the

“overlap” package (Meredith \& Ridout, 2020). Output values range between zero and one, with one

representing complete ITD distribution overlap between species. We only included species with $\geq 10$

individuals that had ITD measured, yielding 45 species pairwise combinations. Additionally, we

performed a mixed-effects ANOVA to test whether bumblebee species identity could significantly

predict mean differences in ITD. Caste was nested within species included as random intercepts, to

ensure that differences between species were not due to one species containing more of a given

caste.

To analyse how ITD varied in space and time at the community level, we first fitted ordinary least squares models, with time expressed as calendar date, and altitude as the mean elevation of each sampling plot. Then, to assess if species responded similarly across time and space, we fitted mixedeffects models (using the "Ime4" package; Bates et al., 2018) and included species as random 
179 likelihood ratio tests (LRT) to determine whether including random intercepts or slopes improved

180 model fit.

\subsection{Assigning individuals to functional groups}

We created individual trait-based functional groups following the method of Rumeu et al. (2018), by

aggregating individual bees based upon similarities in ITD and ignoring species identity (using the

"cluster" package; Maechler et al., 2014). When clustering, we only included flower-visiting bees

that had ITD measurements ( $n=495$; as we use these clusters in later analyses of plant-bumblebee

visitation networks) and performed it on the pooled set of observations across the spatiotemporal

gradient. We "constrained" the number of functional groups to match the total number of species

present, to enable comparisons without confounding effects of changing number of nodes (Rumeu

et al., 2018). The resulting 13 functional groups varied in abundance and ITD range (Fig. S3; Table S6)

and were numbered in order of increasing body size (i.e. Constrained (C) 01 contained the smallestbodied individuals).

To investigate spatial variation in the bumblebee community, we grouped plots from vegetation

types A and B to represent a "low-elevation" dataset, and plots from vegetation types C, D and E, a

"high-elevation" dataset. Pooling data across multiple plots was done to ensure sufficient sample size for our analyses across each point in space and time. Within each spatially pooled set of observations, we split observations into an "early" and "late" part of the season. To guide this split we considered colony-lifecycle phenology of the social bumblebees (queens emerge and dominate during the early season and workers during the late) to be natural stages of comparison to look at temporal functional turnover and determined the cut-off between "early" and "late" as occurring when $50 \%$ of observed female bees were likely to be queens ( $23^{\text {rd }}$ June 2018 ; Table S7; Fig. S4; SI 4). 
202 We estimated spatiotemporal turnover in the taxonomic and functional composition of communities

203 using Bray-Curtis distance matrices (using the "betapart" package; Baselga et al., 2018), which are

204 robust to under-sampling and taxonomic misidentification (Schroeder \& Jenkins, 2018). We also

205 decomposed the Bray-Curtis distances into their two additive components (sensu Baselga, 2013).

206 Bray-Curtis distances range from zero to one, with one representing complete community turnover.

207 To ensure the robustness of our results, we additionally computed Jaccard distances with a

208 presence-absence version of the community data (using "vegan" package; Oksanen et al., 2007), the

209 results of which can be found in the supplementary.

We constructed two types of weighted networks from the same observed interaction data using the

“bipartite" package (Dormann et al., 2009): 1) using species identity as nodes, 2) using functional

group identity as nodes (see 2.5). We described each network using: the number of bumblebee

nodes (taxonomic or functional groups) (B) and plant species (P); and the total number of observed

interactions (I). To investigate whether the functional-group-based networks better capture trait

matching, we additionally calculated: Weighted connectance (herein, connectance; e.g. Kaiser-

Bunbury et al., 2011) to reflect the proportion of realised links in the network out of all possible links

(i.e. $\frac{I}{P \times B}$ ), ranging between zero (unconnected network, suggesting many specialised interactions)

and one (completely connected network); and Weighted quantitative modularity $Q$ (herein,

modularity; Beckett, 2016) to describe the presence of distinct, highly connected subnetworks.

223 (all links are within modules; highly modular network). 
227 vaznull algorithm (Vázquez et al., 2007) from the "bipartite" package (Dormann et al., 2009). These

$228 z$-scores enabled us to estimate the likelihood, as $p$-values, of the observed network architectures

229 emerging from random associations.

2.8 Using stepwise extinctions to compare network robustness between species-and functional-

Through a randomised removal and iteration process we investigated the relative contribution of taxonomic and functional loss towards the rate of network collapse, based on the principle that interaction losses lead to subsequent host-plant extinctions. Here, an extinction occurred when a node had zero links remaining, with the assumption that no interaction rewiring occurred after extinction. By comparing the differential rate of network collapse under functional vs. taxonomic loss, we can investigate which poses a greater threat to plant-pollinator interactions under environmental change, and whether this depends on the spatiotemporal context. We utilised a novel method of stepwise extinction that focuses on the frequency distribution of ITD within species. We removed one discrete portion of a node, where, for the species-based networks, we removed a functional group from a node (e.g. all individuals with ITD 4.04-4.52 $\mathrm{mm}$ being removed from $B$. jonellus), and, for the functional-group-based networks, we removed a species from a node (e.g. $B$. alpinus/polaris was removed from the node containing individuals with 7.71-7.95 mm ITDs). We performed this removal randomly until one of the trophic levels (plant or bumblebee) had only one node remaining. For example, during the late season at low elevation, there are 37 unique species $\times$

246 functional group combinations (number of functional groups within species, or vice versa), meaning

247 a maximum of 37 removals could occur. After each removal, we measured the number of remaining 248 flower species, (weighted) connectance and (weighted quantitative) modularity - we used the rate 249 of change in these metrics as proxies of network robustness. We iterated the process of randomised 250 removal and calculation of indices 1,000 times and performed it separately for each point in space 251 and time. 
252 Using the results from the iterations, we modelled the change in each measure of network

253 robustness as a function of the number of "items" (viz. discrete portions within nodes) that had been

254 removed from each network, using generalised least squares models (gls; from "nlme" package;

255 Pinheiro et al., 2017). Models took either linear or quadratic form, depending on the $\mathrm{R}^{2}$ value. To

256 account for increasing variance of model residuals when items were removed, we included an

257 exponent-of-the-variance covariate. Due to issues with model convergence, we were unable to

258 account for increasing model-residual variance for modularity, so these results have been placed in

259 the supplementary materials, and should be interpreted with caution. To assess and compare levels

260 of "network robustness", we looked at two aspects: i) differences in the shape of the plotted line

261 estimates; ii) area under each curve (AUC) per model ("DescTools” package; Signorell, 2021). We

262 standardised by dividing the raw AUC value by the product of the number of items removed (as

263 some points in space and time contained more functional groups and species) and the maximum

264 value of the calculated index. For the number of remaining plant species, if loss of functional groups

265 has a larger effect than loss of species, we should expect functional-group networks to show lower

266 standardised AUC, as it indicates that fewer individuals have to be removed from the network before

267 its structure collapses. Alternatively, for weighted connectance, a higher AUC when removing

268 functional groups would suggest the network requires fewer individuals to be removed for

269 specialised interactions to be lost (i.e. before it becomes fully connected).

270 We performed all analyses using R (v. 4.0.2; R Core Team, 2020).

\section{RESULTS}

274 Bee species identity did not significantly predict ITD (mixed-effects ANOVA: $F=0.36, p=0.97$; Table

275 S8), with the 13 species showing considerable overlap in ITD distributions (Fig. S5). While bumblebee 
276 ITD ranged from 3.21 to $9.43 \mathrm{~mm}, 80 \%$ of the pairwise species comparisons $(n=45)$ showed

277 overlapping ITD kernel density estimates of $\geq 0.5$, and $11 \%$ showed $\geq 0.75$ (Table S9). B. jonellus and

278 B. pratorum, however, frequently deviated from this trend, each constituting four (of nine) pairwise

279 comparisons with $<0.5$ overlap.

280 At the community level, mean ITD significantly decreased over the season (Ime: $-0.0491 \mathrm{~mm} \mathrm{day}^{-1} \pm$

0.00251, $t=-19.6, p<0.001 ; N=709 ;$ Fig. 2; Table S10-12) and was consistent across bumblebee

species (likelihood ratio tests (LRT) supporting species having random intercepts (LRT: $p<0.001$ ), but

not random slopes (LRT: $p=1$ ) for the effect of season). ITD also significantly increased with

elevation (Im: $0.121 \mathrm{~mm} 100 \mathrm{~m}^{-1} \pm 0.0279, t=4.33, p<0.001 ; N=709$; Fig. 2; Table S13), with species

having different intercepts (LRT: $p<0.001$; Table S14-15) but similar slopes (LRT: $p=0.195$ ).

\subsubsection{Changes in species versus functional composition in the community}

Species turnover was similar in time and space (Bray-Curtis distance $\left(D_{B C}\right): 0.474-0.672 \& 0.322-$

0.781, respectively; Fig. 3; see Table S16-18 for breakdown in the additive components of $D_{B C}$ and

Jaccard dissimilarity). Functional turnover, however, was larger than species turnover (mean $D_{\mathrm{BC}} \pm$

standard error: $0.651 \pm 0.099$ vs. $0.537 \pm 0.068$, respectively; Table S17). As suggested by 3.1 .1 and

Fig. 2, functional turnover was also greater over the season than with elevation $\left(D_{B C}: 0.776-0.792 \&\right.$

0.269-0.500, respectively). Over the season, we saw an increased relative abundance of functional

groups containing smaller individuals: C02 (mean ITD = 3.82 mm), C03 (4.27 mm) and C04 (4.71 mm),

and decrease in the larger-bodied functional groups: $\operatorname{Co7}(6.23 \mathrm{~mm})$ and $\operatorname{Co8}(6.69 \mathrm{~mm})$. With

elevation, groups $\mathrm{C09}(7.22 \mathrm{~mm})$ and $\mathrm{C} 10(7.81 \mathrm{~mm})$ increased in relative abundance, while instead

C03 decreased.

Connectance and modularity differed in the two network types across space and time (Fig. 4), with 
300

301

302

303

304

305

under the null distribution (species (s): late season, low elevation connectance: $z=-3.82, p=<0.001$, modularity: $z=5.17, p<0.001$; late, high connectance: $z=-2.02, p=0.043$, modularity $=z=4.98, p$ $<0.001$; functional (f): late, high connectance: $z=-3.04, p=0.002$; Table S19). During the late season, species-based networks were also consistently more modular than functional-group-based networks (low: $s=0.241, f=0.136$; high: $s=0.334, f=0.245$ ), although both showed similar levels of connectance (Table S19).

\subsection{Differences in robustness between species and functional networks over space and time}

For the stepwise removal analysis, removing functional groups from species nodes always led to faster extinction of flower species $(f=0.738-0.828, s=0.765-0.838$; Fig. $5 \mathrm{~A}$; Tables $\mathrm{S} 20-21)$ than removal of bumblebee species. For flower species, this difference was most pronounced during the early season at high elevation, in which removing species from functional-group nodes showed a convex decline in number of flower species remaining, whereas removing functional groups from species nodes had a linear decline (AUCs: 0.800 vs. 0.738 , respectively). For connectance, the differential rates at which the networks became fully connected under functional vs. taxonomic loss was spatiotemporally dependent. For example, at low elevation, and at high elevation during the early season, removing species from functional group nodes led to a faster loss of specialised interactions than removing functional groups from species nodes (i.e. higher standardised AUCS reflecting a more rapid increase in connectance; Fig. 5B; AUC range: $0.751-0.888$ vs. $0.732-0.885$, respectively). In contrast, at high elevation during the late season, this trend was reversed, with functional loss leading to a faster decline in specialised interactions ( 0.692 vs. 0.764 , respectively).

\section{DISCUSSION}

\subsection{Mapping spatiotemporal trait change reveals decoupling of functional and species}

turnover 
323 When considering the bumblebee community as a static entity, most bumblebee species overlapped

324 in body size suggesting high functional redundancy. Species identity was thus a poor surrogate for

325 individual trait variation. Additionally, body size within a species varied in space and time, such that

326 functional turnover as the season progressed was far larger relative species turnover. While body

327 size turnover in time is somewhat expected in bumblebees (large queens followed by smaller

328 workers), we also found functional turnover in space, which again exceeded species turnover,

329 particularly early in the season. This decoupling of species identities and their mean traits (Robroek

330 et al., 2017) highlights why overlooking the spatiotemporal dynamics of networks, as well as

331 assigning mean trait values to species, may poorly explain functional outcomes such as trait

332 compatibility and resource acquisition (Rumeu et al., 2018; Wong \& Carmona, 2021).

Spatial turnover being greater in functional than in taxonomic communities suggests that, while

species adaptations may have contributed to higher altitude distributions (larger bodied species

found at higher elevations, particularly in early season), intraspecific variation in body size still

mediated where bees could be found. Smaller bees of any species were filtered from the community

at high altitude (also see McCabe et al., 2019). This likely represents a plastic response at the colony

level (Classen et al., 2017), where larger individuals within a species are able to forage at higher

elevation, due to being better able to thermoregulate (Heinrich, 1975) and fly at lower temperatures

340 (Kenna et al., 2021).

341 Temporal functional turnover being independent from species identity is not exclusive to

342 bumblebees (Fukami et al., 2005; Classen et al., 2017; Robroek et al., 2017; Gómez et al., 2020),

343 although the degree of such decoupling may vary across organisms. Yet, surprisingly little work has

344 been done on mapping temporal trait distributions, especially at the spatiotemporal resolution or

345 across a large community that we cover (but see Classen et al., 2017). Further, our study is one of

346 the first to associate such a trait distribution with a key functional role - floral visitation as a proxy

347 for pollination service (but see Ranta \& Lundberg, 1981; Miller-Struttmann \& Galen, 2014; Classen et 
al., 2017). Studies should therefore benefit from considering species as nested within a functional

continuum, rather than as distinct units in terms of their traits (Bolnick et al., 2011; Siefert et al.,

2015; Des Roches et al., 2018).

\subsection{Architecture of functional interactions varied in space and time}

Our plant-bumblebee interaction networks varied architecturally across space and time, contrasting

with previously studied networks that showed relatively stable macroscopic features (e.g.

connectance) across space (at local and regional scales) and over time (within seasons and across

years; Olesen et al., 2008; Dupont et al., 2009; Trøjelsgaard \& Olesen, 2016; Tylianakis \& Morris,

2017). We found modularity to increase and connectance to decrease as the season progressed. This

was associated with an increase in bumblebee abundance and plant species richness, which could

have led to greater competition between bumblebee species promoting more specialised host-plant

interactions (Brosi \& Briggs, 2013; Miller-Struttmann \& Galen, 2014). This is consistent with

ecological character-displacement theory, in which competition leads to niche divergence in

sympatric species; (Germain et al., 2018; Egas et al., 2005). Alternatively, other temporal changes in

our studied plant-pollinator community in association with abiotic factors, like increasing mean daily

temperatures, may have contributed. For example, Ohler et al. (2020) observed that microclimatic

variation in temperature (of soils) was positively related to plant productivity and plant-visitor

richness, and subsequently changes in network architecture. But ultimately, our findings underline

how any static perspective of plant-pollinator interactions will miss nuanced but fundamental

properties of how species spatiotemporally interact.

Functional over taxonomic networks being more connected and less modular later in the season was 
373 2020), clustering of individuals based on their position in multidimensional trait space (Dehling et al.,

374 2016; Coux et al., 2016), and/or measures of evolutionary history (Bascompte et al., 2019; Hurtado

375 et al., 2020) might improve investigations of how trait compatibility determines the spatiotemporal

376 dynamics of interactive networks.

When simulating bumblebee extinction, removing functional groups from networks led to a faster secondary coextinction of plant species than removing species, especially during the early season at high elevation. This is explained by our functional networks being more connected and less modular, enabling perturbations (species loss) to spread quickly (Kortsch et al., 2015). Functional changes to communities, such as declines in body size, have been shown to accompany environmental change drivers (e.g. Guthrie, 2003; Rode et al., 2010; Wu et al., 2019). Our results suggest that functional group loss may cause faster extinction of interaction partners than based on species loss alone especially given body size overlap was as large within as it was between species. It is plausible to lose whole functional groups, for example, if an early drought disproportionately affected larger bumblebees through reduced floral resources (Couvillon \& Dornhaus, 2010) or if a late frost disproportionately affected smaller bees (Heinrich, 1975; Kenna et al., 2021). Studies focusing solely on species loss when investigating how communities may be affected by environmental change (e.g. Burkle et al., 2013; Bascompte et al., 2019; Soroye et al., 2020) may thus underestimate the vulnerability of communities to perturbations.

Bumblebee species removal accelerated the loss of specialised bumblebee-plant interactions relative to functional removal, except during the late season at high elevation, where the reverse occurred. These results highlight the importance of understanding how redundancy varies in time and space. Similarly, Wardle \& Zackrisson (2005) found that empirical removal of species or functional groups affected ecosystem-level properties of some islands (in northern Sweden) but not others, depending on historical disturbance regime, island size and successional stage. The spatiotemporal context 
dependency of community interactions is thus important to consider when understanding network

robustness (Thuiller et al., 2014; Robroek et al., 2017).

Our stepwise modelling approach assumed bumblebee group extinctions could occur randomly.

Drivers of extinctions however likely occur and act non-randomly, such as land-use and climate

change (Larsen et al., 2005), and thus informing extinction order probabilities will give new insights

this is unlikely in real communities (Brosi \& Briggs, 2013; Bramon Mora et al., 2020). However, the studies to collect trait and interaction data at fine spatiotemporal scales to elucidate mutualist and competitive responses to environmental change.

\section{CONCLUSION}

417 Our study reinforces why individual traits should be used to construct interaction networks, and the

418 fundamental importance of where and when networks are studied during a season. The impact of

419 functional or taxonomic losses on network robustness was not uniform in space and time, with the

420 rates of plant coextinctions and loss of specialised interactions varying. However, losing bumblebee

421 functional groups typically accelerated plant coextinctions more than losing species, meaning

422 coextinctions of interaction partners could happen more quickly under functional loss than expected 
423 based on pollinator species extinction. Specifically for our study system, the loss of key functional

424 groups dominating the early season and high altitude could be devastating for the whole plant-

425 pollinator community. Overall, the level of redundancy within plant-pollinator community

426 interactions and subsequently network robustness to perturbation is spatiotemporally dependent.

427 Understanding how trait distributions change in localised space and time is thus essential to

428 understanding dynamic population responses to environmental change.

\section{REFERENCES}

Adams, D.C., Collyer, M.L. \& Kaliontzopoulou, A. (2020). Geomorph: Software for geometric morphometric analyses. R package version 3.2.1.

Bartomeus, I. (2013). Understanding Linkage Rules in Plant-Pollinator Networks by Using Hierarchical Models That Incorporate Pollinator Detectability and Plant Traits. PLoS One, 8, e69200.

Bartomeus, I., Gravel, D., Tylianakis, J.M., Aizen, M.A., Dickie, I.A. \& Bernard-Verdier, M. (2016). A

Bascompte, J. \& Jordano, P. (2007). Plant-Animal Mutualistic Networks: The Architecture of 
446

447

448

449

Bates, D., Maechler, M., Bolker, B., Walker, S., Christensen, R.H.B., Singmann, H., et al. (2018). Package 'Ime4.' Version, 1, 437.

Beckett, S.J. (2016). Improved community detection in weighted bipartite networks. R. Soc. Open Sci., 3.

Bewick, S., Brosi, B.J. \& Armsworth, P.R. (2013). Predicting the effect of competition on secondary plant extinctions in plant-pollinator networks. Oikos, 122, 1710-1719.

Bladon, A.J., Lewis, M., Bladon, E.K., Buckton, S.J., Corbett, S., Ewing, S.R., et al. (2020). How butterflies keep their cool: Physical and ecological traits influence thermoregulatory ability and population trends. J. Anim. Ecol., 89, 2440-2450.

Bolnick, D.I., Amarasekare, P., Araújo, M.S., Bürger, R., Levine, J.M., Novak, M., et al. (2011). Why intraspecific trait variation matters in community ecology. Trends Ecol. Evol.

Boyle, M.J.W., Bishop, T.R., Luke, S.H., van Breugel, M., Evans, T.A., Pfeifer, M., et al. (2020). Localised climate change defines ant communities in human-modified tropical landscapes. Funct. Ecol., 7, 14.

Bramon Mora, B., Shin, E., CaraDonna, P.J. \& Stouffer, D.B. (2020). Untangling the seasonal dynamics of plant-pollinator communities. Nat. Commun., 11, 1-9.

Brosi, B.J. \& Briggs, H.M. (2013). Single pollinator species losses reduce floral fidelity and plant reproductive function. Proc. Natl. Acad. Sci. U. S. A., 110, 13044-13048.

Burkle, L.A., Marlin, J.C. \& Knight, T.M. (2013). Plant-pollinator interactions over 120 years: Loss of species, co-occurrence, and function. Science (80-. )., 340, 1611-1615.

Cane, J.H. (1987). Estimation of Bee Size Using Intertegular Span (Apoidea). J. Kansas Entomol. Soc., $60,145-147$.

CaraDonna, P.J., Petry, W.K., Brennan, R.M., Cunningham, J.L., Bronstein, J.L., Waser, N.M., et al. 
CaraDonna, P.J. \& Waser, N.M. (2020). Temporal flexibility in the structure of plant-pollinator interaction networks. Oikos, 129, 1369-1380.

Cariveau, D.P., Nayak, G.K., Bartomeus, I., Zientek, J., Ascher, J.S., Gibbs, J., et al. (2016). The Allometry of Bee Proboscis Length and Its Uses in Ecology. PLoS One, 11, e0151482.

Couvillon, M.J. \& Dornhaus, A. (2010). Small worker bumble bees (Bombus impatiens) are hardier

Classen, A., Steffan-Dewenter, I., Kindeketa, W.J. \& Peters, M.K. (2017). Integrating intraspecific variation in community ecology unifies theories on body size shifts along climatic gradients. Funct. Ecol., 31, 768-777.

Dongmo, M.A.K., Bonebrake, T.C., Hanna, R. \& Fomena, A. (2018). Seasonal Polyphenism in Bicyclus dorothea (Lepidoptera: Nymphalidae) Across Different Habitats in Cameroon. Environ. Entomol., 47, 1601-1608. against starvation than their larger sisters. Insectes Sociaux, 57, 193-197.

Coux, C., Rader, R., Bartomeus, I. \& Tylianakis, J.M. (2016). Linking species functional roles to their network roles. Ecol. Lett., 19, 762-770.

Dehling, D.M., Jordano, P., Schaefer, H.M., Böhning-Gaese, K. \& Schleuning, M. (2016). Morphology predicts species' functional roles and their degree of specialization in plant-Frugivore interactions. Proc. R. Soc. B Biol. Sci., 283.

Dormann, C.F., Frund, J., Bluthgen, N. \& Gruber, B. (2009). Indices, Graphs and Null Models: 
Dupont, Y.L., Padrón, B., Olesen, J.M. \& Petanidou, T. (2009). Spatio-temporal variation in the structure of pollination networks. Oikos, 118, 1261-1269.

Dupont, Y.L., Trøjelsgaard, K., Hagen, M., Henriksen, M. V., Olesen, J.M., Pedersen, N.M.E., et al.

Eklöf, A., Jacob, U., Kopp, J., Bosch, J., Castro-Urgal, R., Chacoff, N.P., et al. (2013). The dimensionality of ecological networks. Ecol. Lett., 16, 577-583.

Fenberg, P.B., Self, A., Stewart, J.R., Wilson, R.J. \& Brooks, S.J. (2016). Exploring the universal

Fric, Z.K. \& Konvicka, M. (2002). Generations of the polyphenic butterfly Araschnia levana differ in body design. Evol. Ecol. Res. Evolutionary Ecology, Ltd.

Fries, T.C.E. (1925). The vertical distribution of some plants on Nuolja (Torne Lappmark). Bot. Not., $1925,205-216$.

Fukami, T., Martijn Bezemer, T., Mortimer, S.R. \& van der Putten, W.H. (2005). Species divergence

Gentile, G., Bonelli, S. \& Riva, F. (2021). Evaluating intraspecific variation in insect trait analysis. Ecol. and trait convergence in experimental plant community assembly. Ecol. Lett., 8, 1283-1290. Entomol., 46, 11-18. 
Gillespie, M.A.K., Birkemoe, T. \& Sverdrup-Thygeson, A. (2017). Interactions between body size,

Gómez, J.M., Perfectti, F., Armas, C., Narbona, E., González-Megías, A., Navarro, L., et al. (2020).

Gravel, D., Baiser, B., Dunne, J.A., Kopelke, J.-P., Martinez, N.D., Nyman, T., et al. (2019). Bringing

Gray, R.E.J., Ewers, R.M., Boyle, M.J.W., Chung, A.Y.C. \& Gill, R.J. (2018). Effect of tropical forest

Greenleaf, S.S., Williams, N.M., Winfree, R. \& Kremen, C. (2007). Bee foraging ranges and their disturbance on the competitive interactions within a diverse ant community. Sci. Rep., 8, 1-12.

Guthrie, RD. (2003). Rapid body size decline in Alaskan Pleistocene horses before extinction. Nat., relationship to body size. Oecologia, 153, 589-596.

Günter, F., Beaulieu, M., Brunetti, M., Lange, L., Schmitz Ornés, A. \& Fischer, K. (2019). Latitudinal and altitudinal variation in ecologically important traits in a widespread butterfly. Biol. J. Linn.

Gutiérrez, D. \& Wilson, R.J. (2021). Intra- and interspecific variation in the responses of insect 
Heinrich, B. (1975). Thermoregulation in Bumblebees II. Energetics of Warm-up and Free Flight. J. comp. Physiol. Springer-Verlag.

Horne, C.R., Hirst, A.G. \& Atkinson, D. (2015). Temperature-size responses match latitudinal-size Lett., 18, 327-335.

Hurtado, P., Prieto, M., Martínez-Vilalta, J., Giordani, P., Aragón, G., López-Angulo, J., et al. (2020). Disentangling functional trait variation and covariation in epiphytic lichens along a continentwide latitudinal gradient. Proc. R. Soc. B Biol. Sci., 287.

Ings, T.C., Montoya, J.M., Bascompte, J., Blüthgen, N., Brown, L., Dormann, C.F., et al. (2009). Review: Ecological networks - beyond food webs. J. Anim. Ecol., 78, 253-269.

Kaiser-Bunbury, C.N., Valentin, T., Mougal, J., Matatiken, D. \& Ghazoul, J. (2011). The tolerance of

Kenna, D., Pawar, S. \& Gill, R.J. (2021). Thermal flight performance reveals impact of warming on island plant-pollinator networks to alien plants. J. Ecol., 99, 202-213.

Keyes, A.A., McLaughlin, J.P., Barner, A.K. \& Dee, L.E. (2021). An ecological network approach to predict ecosystem service vulnerability to species losses. Nat. Commun., 12, 1-11. 
structure of arctic marine food webs due to poleward shifts of boreal generalists. Proc. R. Soc. B Biol. Sci., 282.

Kuppler, J., Höfers, M.K., Wiesmann, L. \& Junker, R.R. (2016). Time-invariant differences between plant individuals in interactions with arthropods correlate with intraspecific variation in plant phenology, morphology and floral scent. New Phytol., 210, 1357-1368.

Larsen, T.H., Williams, N.M. \& Kremen, C. (2005). Extinction order and altered community structure rapidly disrupt ecosystem functioning. Ecol. Lett., 8, 538-547.

Lecoq, L., Ernoult, A. \& Mony, C. (2021). Past landscape structure drives the functional assemblages of plants and birds. Sci. Rep., 11, 3443.

Lundberg, H. \& Ranta, E. (1980). Habitat and Food Utilization in a Subarctic Bumblebee Community. Oikos, 35, 303.

MacDougall, A.S., Caplat, P., Olofsson, J., Siewert, M.B., Bonner, C., Esch, E., et al. (2021).

Maechler, M., Rousseeuw, P., Struyf, A., Hubert, M., Hornik, K., Studer, M., et al. (2014). Package "cluster."

McCabe, L.M., Cobb, N.S. \& Butterfield, B.J. (2019). Environmental filtering of body size and darker coloration in pollinator communities indicate thermal restrictions on bees, but not flies, at high functional traits. Trends Ecol. Evol., 21, 178-185.

Meredith, M. \& Ridout, M. (2020). Overview of the overlap package. 
Miller-Struttmann, N.E., Geib, J.C., Franklin, J.D., Kevan, P.G., Holdo, R.M., Ebert-May, D., et al. Science (80-. )., 349, 1541-1544.

Ohler, L.-M., Lechleitner, M. \& Junker, R.R. (2020). Microclimatic effects on alpine plant communities and flower-visitor interactions. Sci. Rep., 10, 1-9.

Olesen, J.M., Bascompte, J., Elberling, H. \& Jordano, P. (2008). TEMPORAL DYNAMICS IN A

Oksanen, J., Kindt, R., Legendre, P., O'Hara, B., Stevens, M.H.H., Oksanen, M.J., et al. (2007). The vegan package. Community Ecol. Packag., 10, 719.

Pellissier, L., Albouy, C., Bascompte, J., Farwig, N., Graham, C., Loreau, M., et al. (2018). Comparing species interaction networks along environmental gradients. Biol. Rev., 93, 785-800.

Pigot, A.L., Sheard, C., Miller, E.T., Bregman, T.P., Freeman, B.G., Roll, U., et al. (2020). Macroevolutionary convergence connects morphological form to ecological function in birds.

Pinheiro, J., Bates, D., DebRoy, S., Sarkar, D., Heisterkamp, S., Van Willigen, B., et al. (2017). Package 'nlme.' Linear nonlinear Mix. Eff. Model. version, 3. 
networks in ecology?

R Core Team (2020). R: A language and environment for statistical computing. R Foundation for Statistical Computing, Vienna, Austria. URL https://www.R-project.org/.

Ranta, E. \& Lundberg, H. (1981). Resource utilization by bumblebee queens, workers and males in a subarctic area. Ecography (Cop.)., 4, 145-154.

Ridout, M.S. \& Linkie, M. (2009). Estimating overlap of daily activity patterns from camera trap data. J. Agric. Biol. Environ. Stat., 14, 322-337.

Robroek, B.J.M., Jassey, V.E.J., Payne, R.J., Martí, M., Bragazza, L., Bleeker, A., et al. (2017).

Des Roches, S., Post, D.M., Turley, N.E., Bailey, J.K., Hendry, A.P., Kinnison, M.T., et al. (2018). The

Rode, K.D., Amstrup, S.C. \& Regehr, E. V. (2010). Reduced body size and cub recruitment in polar bears associated with sea ice decline. Ecol. Appl., 20, 768-782.

Rohlf, F.J. (2015). The tps series of software. Hystrix, 26, 1-4.

Rumeu, B., Sheath, D.J., Hawes, J.E. \& Ings, T.C. (2018). Zooming into plant-flower visitor networks: An individual trait-based approach. PeerJ, 2018, e5618.

Schroeder, P.J. \& Jenkins, D.G. (2018). How robust are popular beta diversity indices to sampling error? Ecosphere, 9, e02100. meta-analysis of the relative extent of intraspecific trait variation in plant communities. Ecol. Lett., 18, 1406-1419. 
630 Söderström, B. (2017). Sveriges humlor: en fälthandbok. Entomologiska föreningen i Stockholm.

631 Soroye, P., Newbold, T. \& Kerr, J. (2020). Climate change contributes to widespread declines among

632 bumble bees across continents. Science (80)., 367, 685-688.

Stang, M., Klinkhamer, P.G.L., Waser, N.M., Stang, I. \& van der Meijden, E. (2009). Size-specific

Taylor-Cox, E.D., Macgregor, C.J., Corthine, A., Hill, J.K., Hodgson, J.A. \& Saccheri, I.J. (2020). Wing morphological responses to latitude and colonisation in a range expanding butterfly. PeerJ, 8 , e10352.

Thuiller, W., Pironon, S., Psomas, A., Barbet-Massin, M., Jiguet, F., Lavergne, S., et al. (2014). The European functional tree of bird life in the face of global change. Nat. Commun., 5, 1-10.

Trøjelsgaard, K. \& Olesen, J.M. (2016). Ecological networks in motion: micro- and macroscopic

Tur, C., Vigalondo, B., Trøjelsgaard, K., Olesen, J.M. \& Traveset, A. (2014). Downscaling pollenvariability across scales. Funct. Ecol.

Tylianakis, J.M. \& Morris, R.J. (2017). Ecological Networks Across Environmental Gradients. Annu. abundance and asymmetric interaction strength in ecological networks. Oikos, 116, 1120-1127.

Wardle, D.A. \& Zackrisson, O. (2005). Effects of species and functional group loss on island ecosystem properties. Nat. 2005 4357043, 435, 806-810. 
654 Wong, M.K.L. \& Carmona, C.P. (2021). Including intraspecific trait variability to avoid distortion of
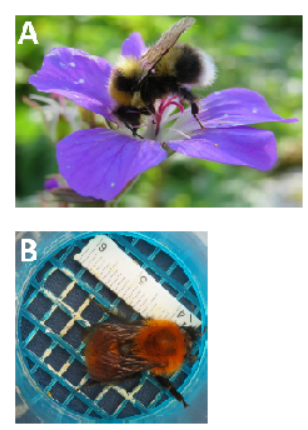

\section{Arctic dwarf shrub heath}

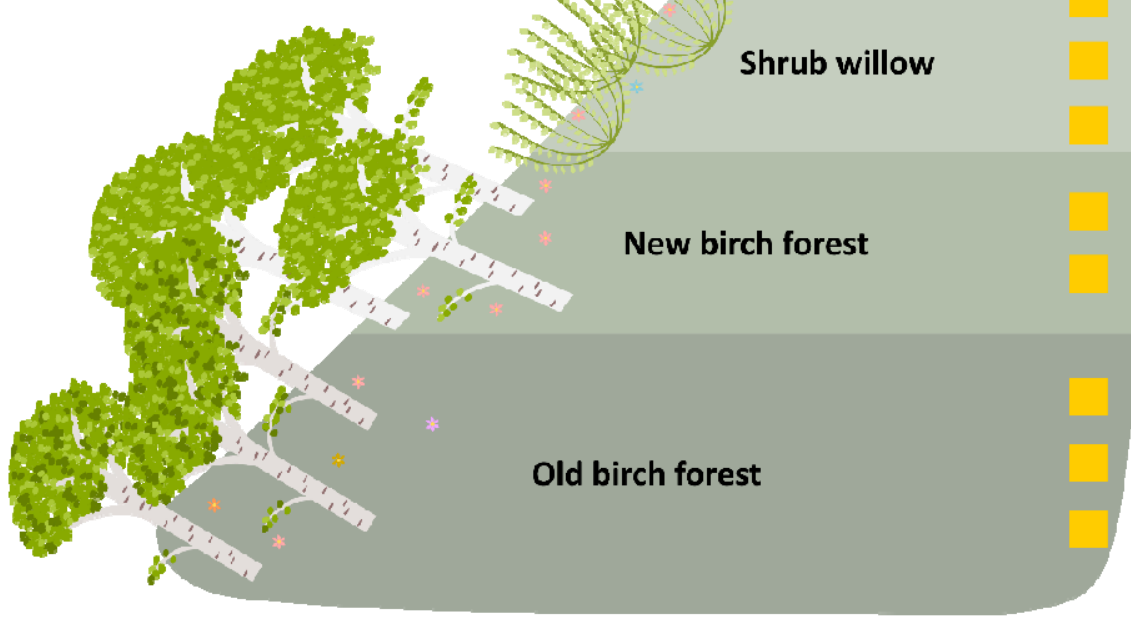

1,164

1,060

\section{Herbaceous} meadow

850

650

550

420

m.a.s.l.

662 Figure 1: The elevational transect on Mount Nuolja spans five vegetation zones with 13 sampling 663 plots (yellow squares). Diagram not to scale. Inset images show: (A) Bombus jonellus foraging on 664 Geranium sylvaticum; (B) B. pascuorum inside a marking cage for measuring the intertegular 665 distance (photo credits: Olivia Bates, Tara Cox \& Aoife Cantwell-Jones). 
A

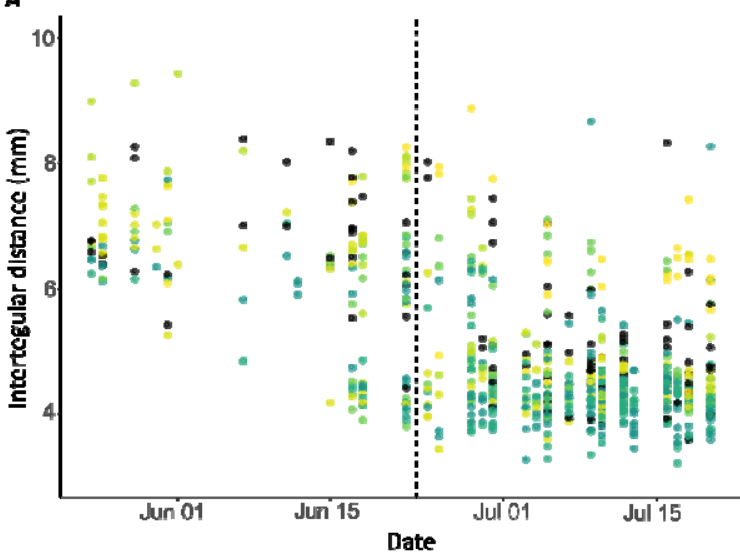

c

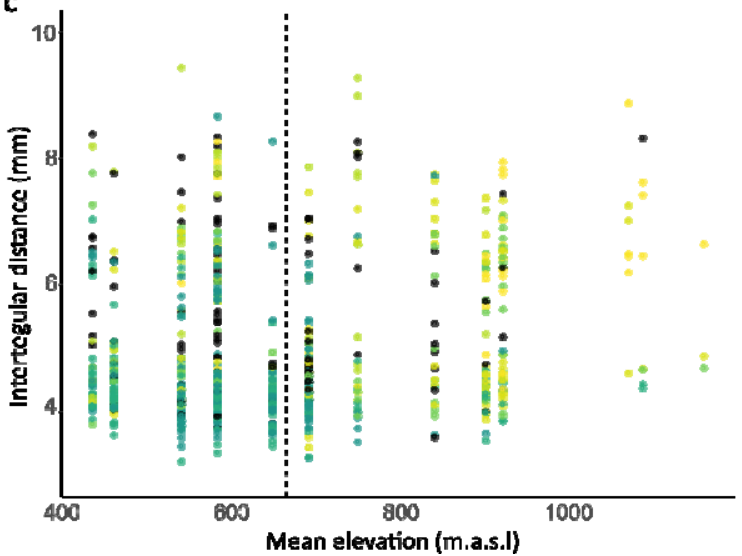

B

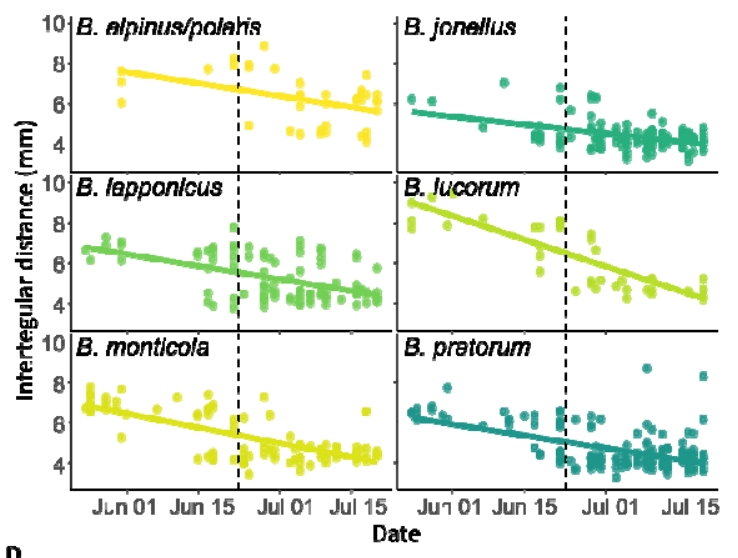

D

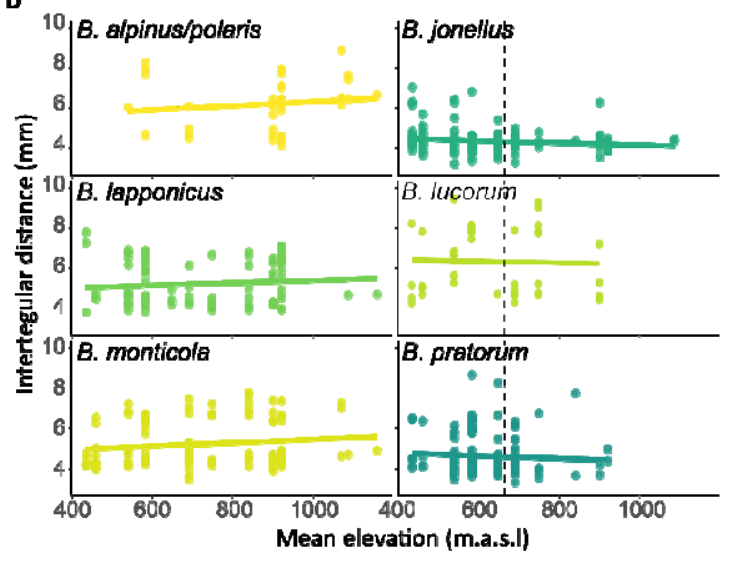

667
Figure 2: Body size (ITD) turnover in space and time for the bumblebee community. Each data point represents a captured individual. Panels A-B show ITD variation as the season progressed at $A$ ) the community level and B) for the six most abundant species, with the dashed line depicting the transition from queen- to worker-dominated foraging, based on estimates from a binomial generalised linear model (Table S7). Panels C-D show ITD variation along the elevational gradient at C) the community level and D) for the six most abundant species, with the dashed line depicting the split between assigned low- (left of line) and high- (right) elevation survey plots. The six most abundant species (Bombus alpinus/polaris, B. jonellus, B. lapponicus, B. lucorum, B. monticola and B. pratorum) are colour coded, with the colours in $A \&$ matching those in $B \& D$. The remaining seven species (B. balteatus, B. bohemicus, B. cingulatus, B. flavidus, B. hortorum, B. hyperboreus and $B$. pascuorum) are black in $A \& C$. 

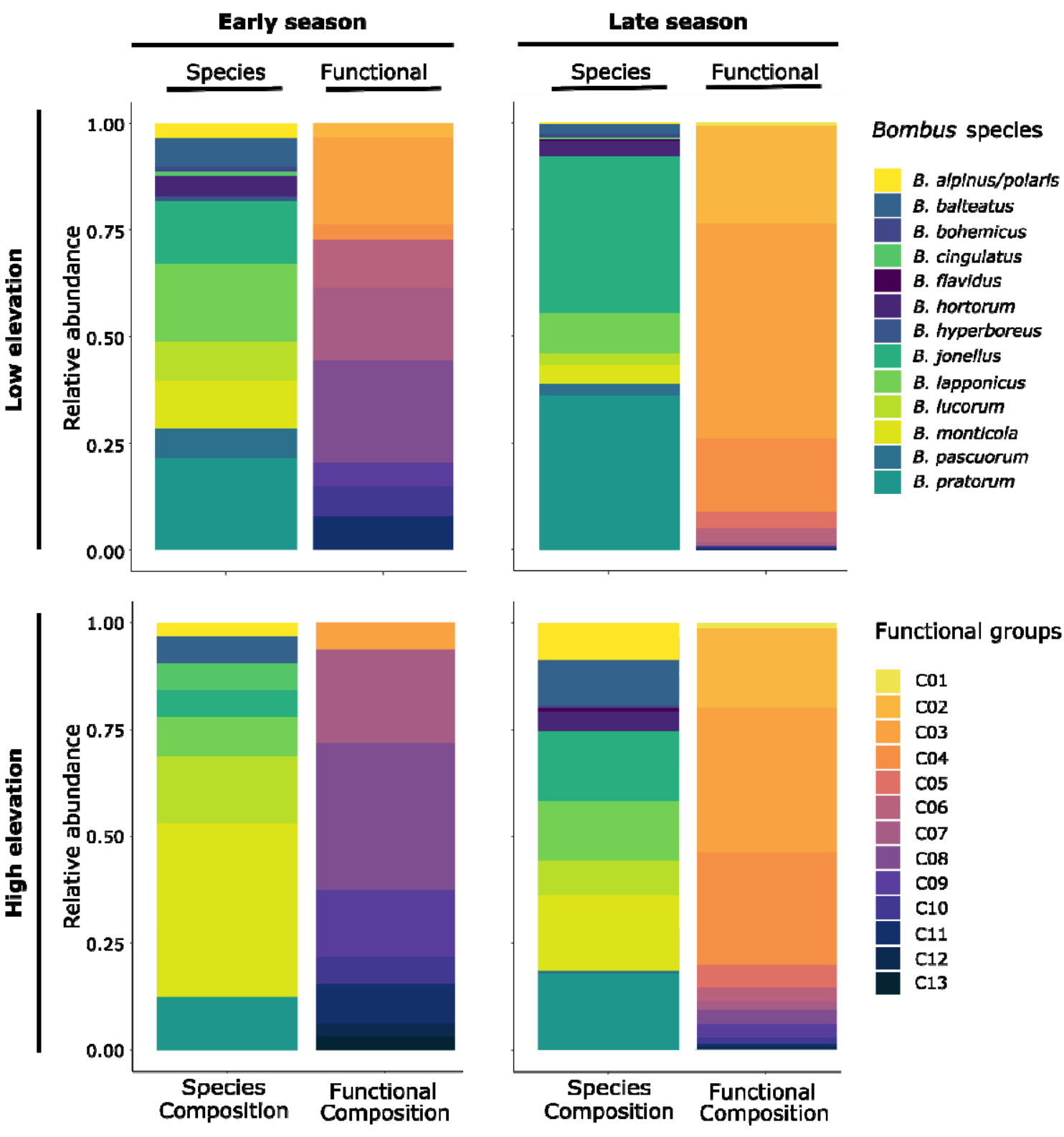

680 Figure 3: Turnover of species- and functional-group-based communities in space (Low \& High 681 elevation) and time (Early \& Late season). The number of individuals in the species- and functional682 group-based bumblebee communities are the same (early season, low elevation $=88$; late, low $=$ 683 224; early, high $=32$; late, high $=151$ ). Functional groups are numbered from the smallest-bodied 684 individuals (C01) to the largest (C13). 

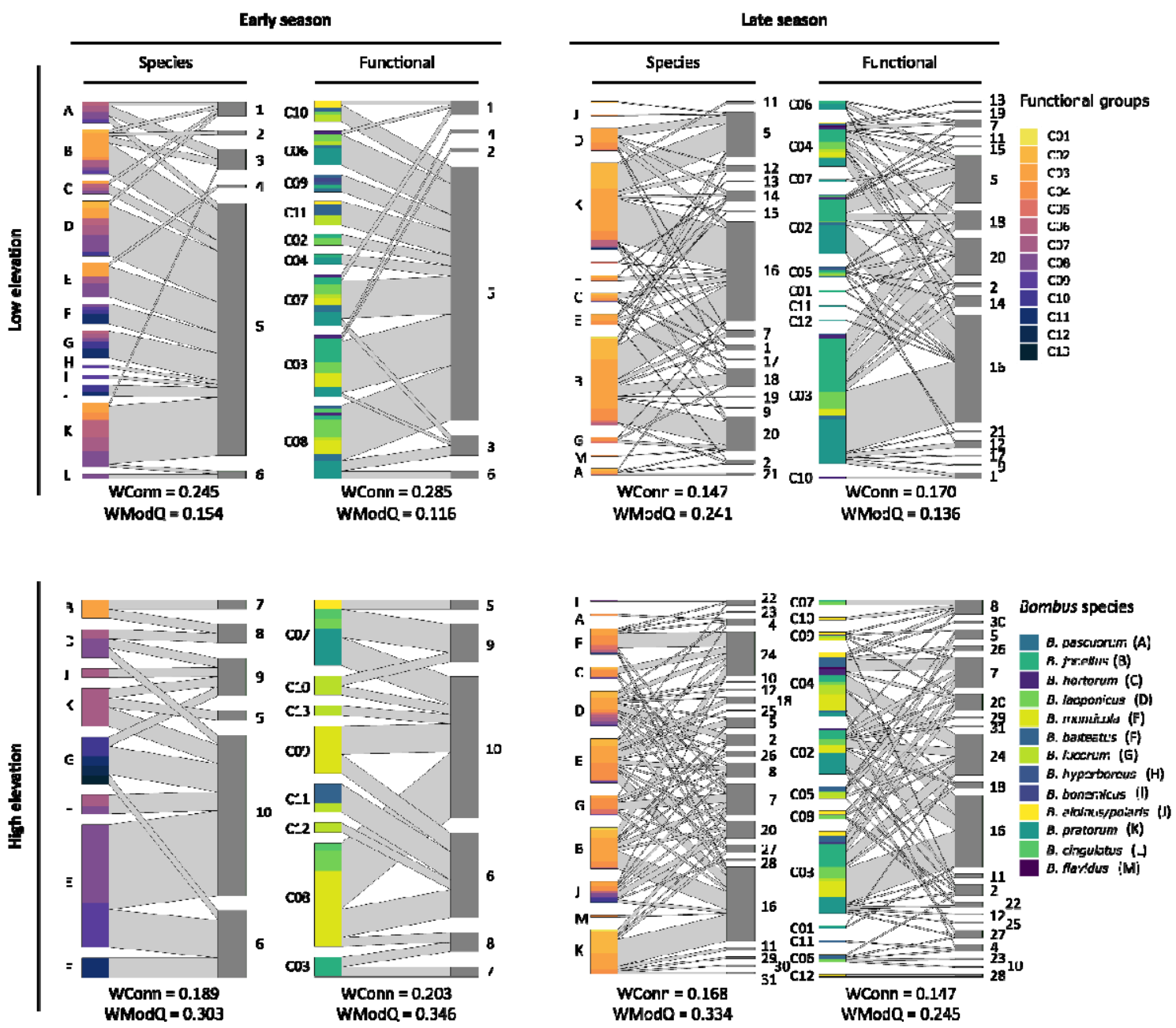

Figure 4: Species- and functional-group-based bumblebee-plant visitation networks at different

points in space and time. Nodes in grey represent plant species: 1) Andromeda polifolia, 2) Pedicularis lapponica, 3) Salix myrsinites, 4) Phyllodoce caerulea, 5) Vaccinium myrtillus, 6) Salix phylicifolia, 7) Trollius europaeus, 8) Salix glauca, 9) Salix myrsinifolia, 10) Salix lanata, 11) Melampyrum sy/vaticum, 12) Solidago virgaurea, 13) Silene dioica, 14) Myosotis decumbens, 15) Rubus saxatilis, 16) Geranium sy/vaticum, 17) Linnaea borealis, 18) Vaccinium uliginosum, 19) Saxifraga aizoides, 20) Vaccinium vitis-idaea, 21) Melampyrum pratense, 22) Taraxacum spp., 23) Ranunculus acris, 24) Astralagus alpinus, 25) Epilobium alsinifolium, 26) Pyrola rotundifolia, 27) Potentilla crantzii, 28) Diapensia lapponica, 29) Bartsia alpina, 30) Pyrola minor, and 31) Viola biflora. For each bipartite network, the coloured nodes on the left trophic level represent bumblebee groups. For networks in the species column, we show the functional group composition of each species indicated by different colours (C01 representing the smallest-bodied bees and $\mathrm{C} 13$ the largest). For the networks in the functional column (where groups of bumblebees are clustered based on ITD similarity), we show the species composition of each functional-group node as indicated by different colours. At the bottom of each network is shown its raw weighted connectance (WConn) and weighted quantitative modularity (WModQ). 

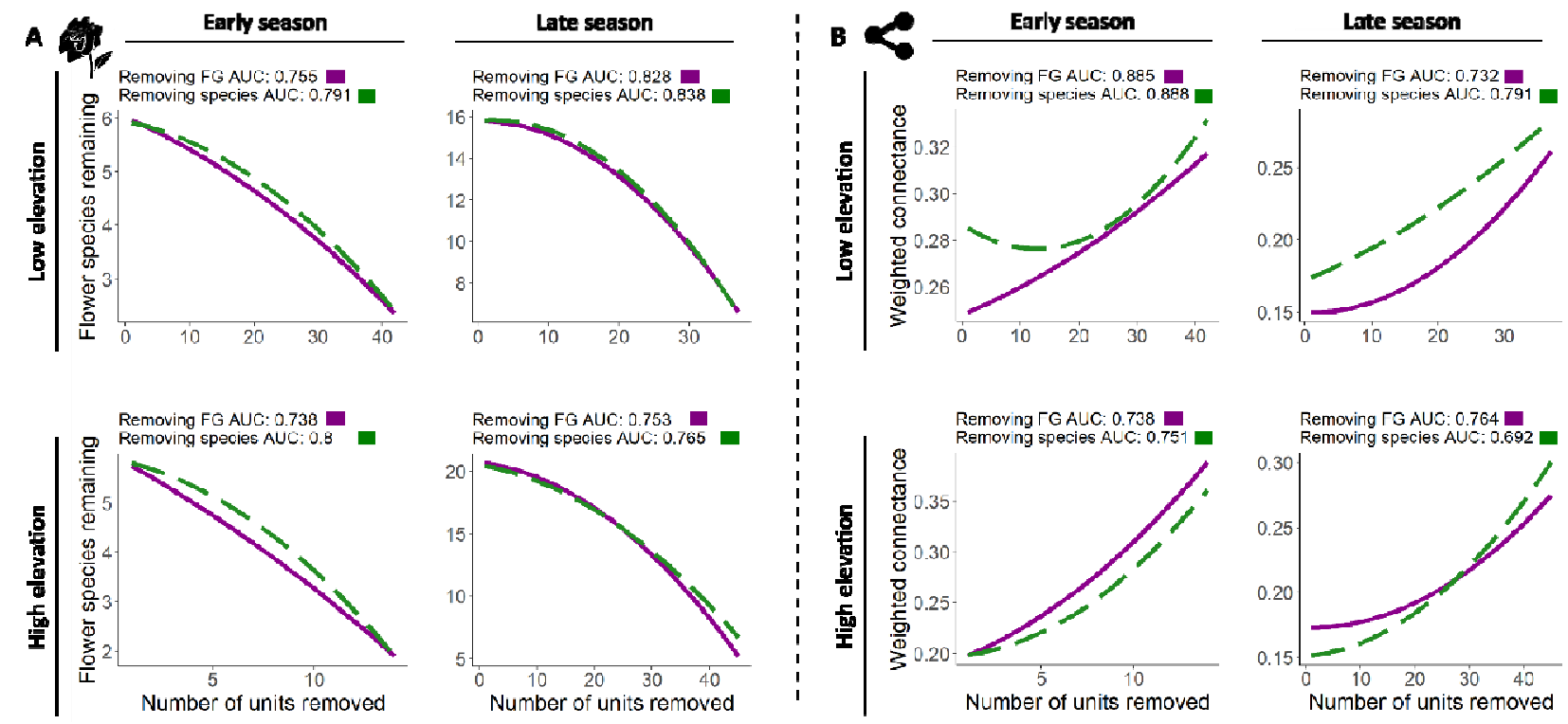

Figure 5: A) Number of remaining flower species and B) change in weighted connectance, in response to stepwise bumblebee removals to assess network robustness to interaction extinctions. Simulations randomly removed either species from functional-group nodes (green dashed line) or functional groups from species nodes (purple solid line), for 1,000 iterations. At each random removal, the number of remaining flower species and weighted connectance were calculated (for graphs with raw values, see Figs. S6-9). Standardised area under the curve (AUC) values are given for each plot. A) Higher AUC suggests slower co-extinction of flower species, i.e. greater robustness to extinction. B) Lower AUC suggests delayed loss of specialised interactions (longer time for network to become fully connected), i.e. greater robustness to extinction. FG =

714 functional groups. 